\title{
Avaliação terapêutica do artesunato na infecção experimental pelo Schistosoma mansoni
}

\author{
Therapeutical evaluation of the artesunate in experimental \\ Schistosoma mansoni infection \\ Neusa Araújo, Anna Kohn e Naftale Katz
}

\begin{abstract}
Resumo Camundongos infectados experimentalmente com Schistosoma mansoni foram tratados, em dose oral única, com artesunato ( $L A C T A B^{\circledR}$ ), 300 ou $500 \mathrm{mg} / \mathrm{kg}$ ou durante cinco dias consecutivos. Os animais foram sacrificados 7, 30, 60 ou 90 dias após o tratamento. Diferenças estatisticamente significativas foram encontradas na distribuição e mortalidade dos vermes e na alteração do oograma nos grupos tratados quando comparados ao controle, em todos os esquemas testados quando os animais foram sacrificados 30 dias após o tratamento. A análise morfológica dos vermes mostrou alterações no aparelho reprodutor feminino com diminuição do volume ovariano, rarefação dos folículos vitelínicos, alterações estas mais acentuadas nas dosagens mais altas, justificando o encontro na alteração do oograma que chegou a 100\%. Entretanto, quando os animais foram sacrificados 60 ou 90 dias após o tratamento, as diferenças e alterações foram menores, mostrando que os vermes sobreviventes se recuperaram e reiniciaram a postura.
\end{abstract}

Palavras-chaves: Schistosoma mansoni. Esquistossomicida. Artesunato.

\begin{abstract}
Mice experimentally infected with Schistosoma mansoni were treated orally with artesunate (Lactab ${ }^{\circledR}$ ) in a single dose of 300 or $500 \mathrm{mg} / \mathrm{kg}$ or over a period of five consecutive days. The animals were sacrificed 7, 30, 60 or 90 days after treatment. Statistically significant differences were found in the distribution and mortality of the worms and in the alterations of the oogram in the treated group when compared to control in all of the tested schemes when the animals were sacrificed 30 days after treatment. Morphological analysis of female worms showed a reduction of ovarian volume and rarefaction of the vitelline follicles. These modifications were more marked after treatment with the higher dose, explaining the alteration of the oogram which reached $100 \%$. However, when the animals were sacrificed 60 or 90 days after treatment, the differences and alterations were smaller, showing that the surviving worms recovered and restarted oviposition.
\end{abstract}

Key-words: Schistosoma mansoni. Schistosomicide. Artesunate.

Centro de Pesquisas René Rachou, Fundação Oswaldo Cruz, MS, Belo Horizonte, MG, Brasil.

Departamento de Helmintologia, Instituto Oswaldo Cruz, Fundação Oswaldo Cruz, Rio de Janeiro, RJ, Brasil.

Auxílio Financeiro FIOCRUZ e CNPq.

Endereço para correspondência: Dra. Neusa Araújo. Centro de Pesquisas René Rachou/FIOCRUZ. Av. Augusto de Lima 1715, 30190-002 Belo Horizonte, MG, Brasil.

Tel: 5531 295-3566; Fax 5531 295-3115.

Recebido para publicação em 13/3/98. 
A artemisinina é um composto isolado da Artemisia annua chamado de Qinghaosu, desde 1972, na China, onde vem sendo usado no tratamento da malária, apresentando resultados satisfatórios 23 . Em 1991, Araújo et al 1 estudaram a atividade do artemether em camundongos e hamsters infectados experimentalmente pelo Schistosoma mansoni. Em camundongos, a droga revelou alta atividade esquistossomicida quando usada em dose única, intramuscular de $100 \mathrm{mg} / \mathrm{kg}$ e uma menor atividade com a mesma dose por via oral. Camundongos tratados com $200 \mathrm{mg} / \mathrm{kg}$, dose única, via intramuscular apresentaram $100 \%$ de alteração do oograma, quando sacrificados 15 dias após o tratamento e oograma normal (ovos em todos os estágios) após 42 dias de tratados. Doses de $100 \mathrm{mg} / \mathrm{kg} / \mathrm{dia}$, intramuscular, aplicadas durante 3 ou 5 dias consecutivos, mostraram 76,6 e $96,3 \%$ de vermes mortos. A análise morfológica dos vermes coletados de camundongos, tratados com a dose única, intramuscular, de $100 \mathrm{mg} / \mathrm{kg}$, não apresentou alterações estruturais significativas que pudessem ser observadas ao microscópio óptico quando os animais foram sacrificados 24 horas após o tratamento. Por outro lado, a análise realizada em camundongos, sacrificados 3 dias após o tratamento, com a mesma dose citada anteriormente, detectou uma diminuição significativa no tamanho dos vermes (machos e fêmeas), alterações degenerativas no parênquima e no sistema reprodutor das fêmeas, com redução do material vitelínico e do volume do ovário e acentuada despigmentação. Nos vermes machos, não foram observadas alterações significativas.

Resultados semelhantes do artemether sobre infecção experimental de $S$. mansoni e $S$. japonicum foram anteriormente publicados 810 .

Entretanto, a baixa solubilidade da artemisinina em água e óleo torna difícil a sua aplicação em larga escala. $\mathrm{O}$ artesunato, um derivado do artemether é solúvel em água e praticamente não apresenta efeito colateral quando administrado por via intramuscular ou intravenosa, usando animais como modelo experimental7, ou na clínica, quando administrado por via intravenosa 4 .

No presente trabalho, são apresentados os resultados obtidos em camundongos infectados experimentalmente com a cepa LE de S. mansoni e tratados com artesunato.

\section{MATERIAL E MÉTODOS}

Camundongos albinos fêmeas, com peso médio de $20 \mathrm{~g}$, foram infectados, por via subcutânea, com $100 \pm 10$ cercárias da cepa LE de S. mansoni.

Quarenta e cinco dias após a infecção, grupos de 15 camundongos cada foram tratados com artesunato (Lactab ${ }^{\circledR}$ ) produzido pelo Laboratório Mepha Ltda. A droga foi administrada por via oral em dose única ou durante cinco dias consecutivos de 300 ou $500 \mathrm{mg} / \mathrm{kg}$ usando as mesmas dosagens do esquema anterior. Os animais foram sacrificados 7, 30, 60 ou 90 dias após o tratamento.

A avaliação da eficácia do artesunato foi baseada na média e na distribuição dos vermes no sistema porta e na percentagem de vermes mortos e de alteração do oograma. Foi considerado alterado o oograma no qual estivessem ausentes um ou mais estágios de desenvolvimento dos ovos6.
Os vermes recuperados por perfusão foram fixados com formol neutro a $10 \%$, corados pelo carmin cloridríco alcoólico, desidratados na série alcoólica, diafanizados com creosoto de Faia e montados em bálsamo do Canadá para análise ao microscópio óptico5.

As fotografias em microscopia de luz foram realizadas em microscópio de laser confocal Zeiss LSM 410 e a leitura com laser de hélio/neônio $(543 \mathrm{~mm})$ sob filtro LP 570.

Paralelamente, aos grupos onde se estudou a eficácia do artesunato foram acompanhados grupos controles de infecção que, à exceção da administração da droga, foram mantidos em condições iguais aos grupos tratados, até aos 45 dias da infecção, quando foram sacrificados.

A avaliação de significância das diferenças encontradas entre os grupos tratados e controles foi feita através do teste de qui-quadrado de Pearson, considerando-se o valor mínimo de significância de $p \leq 0,059$.

\section{RESULTADOS}

Camundongos tratados com artesunato apresentaram diferenças significativas, quando comparados aos grupos controles, nas percentagens de distribuição dos vermes no sistema porta, mortalidade dos mesmos e de alteração do oograma. Com a dose única de 
$300 \mathrm{mg} / \mathrm{kg}$ foram encontradas diferenças significativas na distribuição de vermes quando os camundongos foram sacrificados após 7 ou 30 dias do tratamento, na mortalidade de vermes nos animais sacrificados 30 dias após o tratamento, e na alteração do oograma que foi de 100 e $25 \%$ nos animais sacrificados, respectivamente, 7 e 30 dias após o tratamento e de $0 \%$ no grupo controle. Aos 60 dias após o tratamento, os parâmetros examinados foram semelhantes aos do grupo controle não tratado. Este esquema não permitiu a avaliação da eficácia após 90 dias do tratamento porque não houve sobreviventes (Tabela 1).

Tabela 1 - Atividade esquistossomicida do artesunato (300mg/kg), dose única, via oral em camundongos infectados experimentalmente com Schistosoma mansoni.

\begin{tabular}{|c|c|c|c|c|c|c|c|}
\hline \multirow{2}{*}{$\begin{array}{l}\text { Examinados } \\
\text { após } \\
\text { tratamento (dia) }\end{array}$} & \multicolumn{2}{|c|}{ Animais } & \multirow{2}{*}{$\begin{array}{c}\text { Média } \\
\text { de } \\
\text { vermes }\end{array}$} & \multicolumn{2}{|c|}{ Distribuição de vermes \% } & \multirow{2}{*}{$\begin{array}{c}\text { Vermes } \\
\text { mortos } \\
\text { no fígado \% }\end{array}$} & \multirow{2}{*}{$\begin{array}{c}\text { Alteração } \\
\text { do } \\
\text { oograma \% }\end{array}$} \\
\hline & tratados & examinados & & mesentério & fígado & & \\
\hline 7 & 15 & 11 & 46,4 & $75,3^{*}$ & 24,7 & 5,9 & $100,0^{*}$ \\
\hline 30 & 15 & 8 & 27,9 & $80,7^{*}$ & 19,3 & $14,3^{*}$ & 25,0 \\
\hline 60 & 15 & 5 & 29,6 & 93,9 & 6,1 & 2,7 & 0,0 \\
\hline controle $^{\star \star}$ & - & 6 & 31,5 & 100,0 & 0,0 & 0,0 & 0,0 \\
\hline
\end{tabular}

${ }^{\star} \mathrm{p} \leq 0,05$.

** Animais sacrificados 45 dias após infecção, sem tratamento.

Camundongos tratados com a dose única de $500 \mathrm{mg} / \mathrm{kg}$ mostraram diferenças significativas na distribuição, com deslocamento dos vermes para o fígado e na mortalidade dos vermes, sendo que esta última alcançou um máximo de
$52,1 \%$ nos camundongos sacrificados 30 dias após o tratamento. Houve alteração do oograma em $100 \%$ dos animais sacrificados com 7 dias e em $40 \%$ daqueles sacrificados com 30 e 60 dias de tratados (Tabela 2).

Tabela 2 - Atividade esquistossomicida do artesunato (500mg/kg), dose única, via oral em camundongos infectados experimentalmente com Schistosoma mansoni.

\begin{tabular}{|c|c|c|c|c|c|c|c|}
\hline \multirow{2}{*}{$\begin{array}{l}\text { Examinados } \\
\text { após } \\
\text { tratamento (dia) }\end{array}$} & \multicolumn{2}{|c|}{ Animais } & \multirow{2}{*}{$\begin{array}{c}\text { Média } \\
\text { de } \\
\text { vermes }\end{array}$} & \multicolumn{2}{|c|}{ Distribuição de vermes \% } & \multirow{2}{*}{$\begin{array}{c}\text { Vermes } \\
\text { mortos } \\
\text { no fígado \% }\end{array}$} & \multirow{2}{*}{$\begin{array}{c}\text { Alteração } \\
\text { do } \\
\text { oograma \% }\end{array}$} \\
\hline & tratados & examinados & & mesentério & fígado & & \\
\hline 7 & 15 & 8 & 20,8 & 93,4 & 6,6 & 4,2 & $100,0^{*}$ \\
\hline 30 & 15 & 5 & 9,6 & $47,9^{*}$ & 52,1 & $52,1^{*}$ & $40,0^{*}$ \\
\hline 60 & 15 & 5 & 13,2 & $60,6^{*}$ & 39,4 & $31,8^{*}$ & $40,0^{*}$ \\
\hline 90 & 15 & 3 & 12,0 & 88,9 & 11,1 & 0,0 & 0,0 \\
\hline controle $^{\star *}$ & - & 10 & 20,5 & 84,4 & 15,6 & 0,0 & 0,0 \\
\hline
\end{tabular}

${ }^{\star} \mathrm{p} \leq 0,05$.

** Animais sacrificados 45 dias após infecção, sem tratamento.

A Tabela 3 apresenta os resultados obtidos quando os camundongos foram tratados com $300 \mathrm{mg} / \mathrm{kg}$ durante 5 dias consecutivos sendo observadas diferenças significativas na distribuição e mortalidade dos vermes quando os animais foram sacrificados após 7, 30 ou 60 dias do tratamento e na alteração do oograma que foi de 100 e $33,3 \%$ nos camundongos sacrificados respectivamente com 7 e 30 dias, enquanto o grupo controle não apresentou alteração.

Com a dose mais alta $(500 \mathrm{mg} / \mathrm{kg} \times 5$ dias) foram encontradas diferenças significativas em todos os parâmetros comparados nos animais sacrificados 7, 30 ou 60 dias após o tratamento. A alteração do oograma foi de, respectivamente, 100, 45,5 e 50,0\% (Tabela 4).

Estudos morfológicos nos vermes recuperados 7 dias após o tratamento mostraram alterações no aparelho reprodutor feminino com diminuição do volume ovariano, redução e rarefação dos folículos vitelínicos com alterações celulares. As fêmeas apresentaram também mudanças degenerativas no tegumento do corpo. As alterações foram mais acentuadas com as doses maiores (Figuras 1, 2, 3 e 4). 
A raújo $\mathrm{N}$ et al

Tabela 3 - Atividade esquistossomicida do artesunato (300 $\mathrm{mg} / \mathrm{kg} / \mathrm{dia}$ × 5 dias), dose única, via oral em camundongos infectados experimentalmente com Schistosoma mansoni.

\begin{tabular}{|c|c|c|c|c|c|c|c|}
\hline \multirow{2}{*}{$\begin{array}{l}\text { Examinados } \\
\text { após } \\
\text { tratamento (dia) }\end{array}$} & \multicolumn{2}{|c|}{ Animais } & \multirow{2}{*}{$\begin{array}{l}\text { Média } \\
\text { de } \\
\text { vermes }\end{array}$} & \multicolumn{2}{|c|}{ Distribuição de vermes \% } & \multirow{2}{*}{$\begin{array}{c}\text { Vermes } \\
\text { mortos } \\
\text { no fígado \% }\end{array}$} & \multirow{2}{*}{$\begin{array}{l}\text { Alteração } \\
\text { do } \\
\text { oograma \% }\end{array}$} \\
\hline & tratados & examinados & & mesentério & fígado & & \\
\hline 7 & 15 & 3 & 27,3 & $54,9^{*}$ & 45,1 & $11,0^{\star}$ & $100,0^{*}$ \\
\hline 30 & 15 & 3 & 19,0 & $66,7^{\star}$ & 33,3 & $24,6^{*}$ & 33,3 \\
\hline 60 & 15 & 5 & 20,4 & $64,7^{\star}$ & 35,3 & $19,6^{*}$ & 20,0 \\
\hline 90 & 15 & 3 & 23,4 & 91,5 & 8,5 & 2,8 & 0,0 \\
\hline controle $^{\star *}$ & - & 10 & 20,5 & 84,4 & 15,6 & 0,0 & 0,0 \\
\hline
\end{tabular}

${ }^{*} \mathrm{p} \leq 0,05$.

** Animais sacrificados 45 dias após infecção, sem tratamento.

Tabela 4 - Atividade esquistossomicida do artesunato (500mg/kg/dia x 5 dias), dose única, via oral em camundongos infectados experimentalmente com Schistosoma mansoni.

\begin{tabular}{|c|c|c|c|c|c|c|c|}
\hline \multirow{2}{*}{$\begin{array}{l}\text { Examinados } \\
\text { após } \\
\text { tratamento (dia) }\end{array}$} & \multicolumn{2}{|c|}{ Animais } & \multirow{2}{*}{$\begin{array}{c}\text { Média } \\
\text { de } \\
\text { vermes }\end{array}$} & \multicolumn{2}{|c|}{ Distribuição de vermes \% } & \multirow{2}{*}{$\begin{array}{c}\text { Vermes } \\
\text { mortos } \\
\text { no fígado \% }\end{array}$} & \multirow{2}{*}{$\begin{array}{c}\text { Alteração } \\
\text { do } \\
\text { oograma \% }\end{array}$} \\
\hline & tratados & examinados & & mesentério & fígado & & \\
\hline 7 & 15 & 5 & 22,3 & $68,5^{*}$ & 31,5 & $17,1^{*}$ & $100,0^{*}$ \\
\hline 30 & 15 & 11 & 15,4 & $66,9^{*}$ & 33,1 & $19,5^{\star}$ & $45,5^{\star}$ \\
\hline 60 & 15 & 6 & 18,2 & 74,3 & 25,7 & 8,3 & $50,0^{*}$ \\
\hline 90 & 15 & 3 & 10,7 & 75,0 & 25,0 & 6,3 & 33,3 \\
\hline controle $^{\star \star}$ & - & 10 & 20,5 & 84,4 & 15,6 & 0,0 & 0,0 \\
\hline
\end{tabular}

${ }^{*} p \leq 0,05$

** Animais sacrificados 45 dias após infecção, sem tratamento.
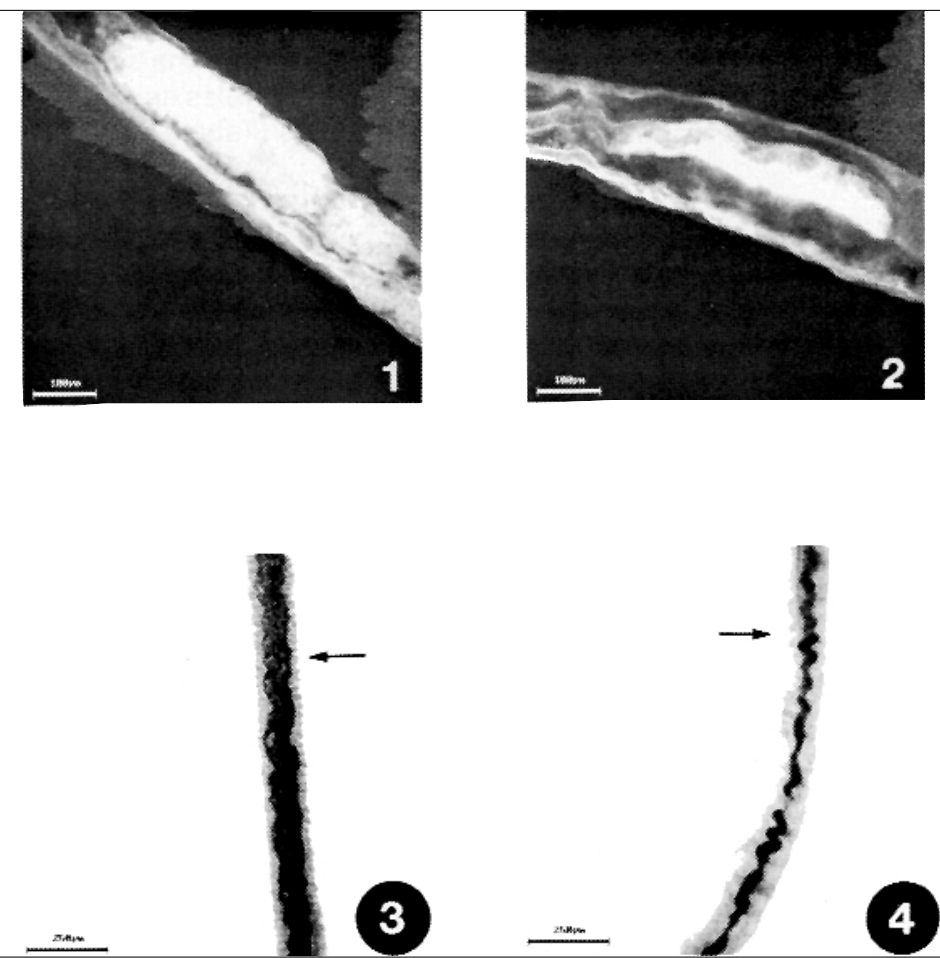

Figura 1 - Ovário de S. mansoni do grupo controle em microscópio laser confocal modo transmitido. Figura 2 - Ovário de S. mansoni (após 7 dias de tratamento com dose única de 500mg/kg de artesunato, via oral), apresentando redução de volume em microscópio laser confocal modo transmitido.

Figura 3 - Folículos vitelínicos de S. mansoni do grupo controle em microscópio laser confocal modo refletido.

Figura 4 - Folículos vitelínicos de S. mansoni (após 7 dias de tratamento com dose única de $500 \mathrm{mg} / \mathrm{kg}$ de artesunato, via oral), apresentando alterações celulares nos folículos vitelínicos e no tegumento, em microscópio laser confocal modo refletido. 


\section{DISCUSSÃO}

Os resultados apresentados mostram que o artesunato age sobre o $S$. mansoni provocando alterações estruturais no aparelho reprodutor das fêmeas, com redução dos folículos vitelínicos resultando, consequentemente, numa parada de postura. Este fato justifica o aparecimento de $100 \%$ de alteração do oograma em alguns esquemas terapêuticos usados.

A dose única de $300 \mathrm{mg} / \mathrm{kg}$, por via oral, causou parada de postura em $100 \%$ dos animais infectados, entretanto a mortalidade dos vermes no fígado foi baixa $(5,9 \%)$. Sessenta dias após o tratamento, as fêmeas já haviam reiniciado a postura o que pode ser comprovado pela inexistência de alteração do oograma. Resultados semelhantes foram observados com a dosagem de $300 \mathrm{mg} / \mathrm{kg}$, por via oral administrados durante cinco dias consecutivos.

O esquema terapêutico de $500 \mathrm{mg} / \mathrm{kg}$, administrado em dose única mostrou que, apesar da percentagem de mortalidade dos vermes ter atingido um nível significativo após 30 e 60 dias do tratamento $(52,1$ e 31,8 , respectivamente) a percentagem de alteração do oograma caiu de $100 \%$ quando os animais foram sacrificados 7 dias após a administração da droga para $40 \%$ após 30 e 60 dias do tratamento. Não foram encontrados vermes mortos no fígado nem alteração do oograma quando os animais foram sacrificados 90 dias após o tratamento. Quinhentos $\mathrm{mg} / \mathrm{kg}$ administrados por 5 dias consecutivos a exemplo dos outros esquemas usados apresentou significativa percentagem de vermes mortos no fígado e $100 \%$ de alteração do oograma quando os animais foram, sacrificados 7 dias após o tratamento. Neste esquema, ao contrário dos anteriores, a alteração do oograma persistiu, em nível significativo, até - 60 ia após o tratamento, e mesmo após 90 dias houve alteração, embora não significativa de $33,3 \%$ do oograma.

Efeitos terapêuticos semelhantes aos alteração do oograma.

AGRADECIMENTOS relatados no presente trabalho foram mostrados por Yue et al10 que estudaram a ação do artemether em camundongos infectados experimentalmente com S. japonicum. Os autores mostraram que 3 a 7 dias após o tratamento com a dose de $100 \mathrm{mg} / \mathrm{kg} / \mathrm{dia} \times 3$ dias, por via subcutânea, $100 \%$ dos vermes migrou para o fígado, e que $74,0 \%$ retornou ao mesentério no $14^{\circ}$ dia após o tratamento. Três a cinco dias após o tratamento ou não foram encontrados ovos no útero das fêmeas ou eles estavam degenerados. Entretanto, ovos normais puderam ser encontrados 11 dias mais tarde, mostrando que uma vez passado o efeito da droga, a infecção seguiu o seu curso normal. Shuhua e Catto 8 estudaram a atividade esquistossomicida do artemether in vitro e in vivo mostrando que a droga é responsável por uma redução de 30 a $50 \%$ no tamanho dos vermes quando observados 14 dias após o tratamento. Porém quando a observação foi feita após 56 dias do tratamento os vermes já se apresentavam com seu tamanho normal. Alterações, também reversíveis, foram encontradas nos testículos e ovários dos vermes, quando foi administrada dose única, oral de $300 \mathrm{mg} / \mathrm{kg}$. Araújo et al 1 mostraram que $100 \mathrm{mg} / \mathrm{kg}$ de artemether, em dose única, por via intramuscular causou parada de postura em $100 \%$ dos animais infectados com S. mansoni, quando a avaliação foi feita com 15 dias. Entretanto, este efeito durou menos que 45 dias quando não mais foram encontradas alterações no oograma. A mesma dose administrada por via oral mostrou somente $22,6 \%$ de vermes mortos, sem apresentar alteração do oograma.

Os dados ora apresentados revelam atividade parcial do artesunato sobre o S. mansoni, com alteração morfológica dos vermes e parada de postura, efeitos estes que se mostraram dose-dependentes, pois passado o efeito da droga, houve a recidiva, que foi constatada pelo desaparecimento das alterações morfológicas dos vermes e pela ausência de
Os autores agradecem ao Dr. Henrique Lenzi, Chefe do Departamento de Patologia do Instituto

Oswaldo Cruz e a Marcelo Pelajo Machado e Bruno Silva Vale, pelas fotografias no microscópio confocal.

\section{REFERÊNCIAS BIBLIOGRÁFICAS}

1. Araújo N, Kohn A, Katz N. Activity of the artemether in experimental Schistosomiasis mansoni. Memórias do Instituto Oswaldo Cruz 86 (supl II): 185-188, 1991. 
2. China Cooperative Research Group on Qinghaosu and its Derivatives as Antimalarials. Antimalarial efficacy and mode of action of Qinghaosu and its derivatives in experimental models. Journal Traditional Chinese Medicine 2: 17-24, 1982a.

3. China Cooperative Research Group on Qinghaosu and its Derivatives as Antimalarials. Studies on the toxicity of Qinghaosu and its derivatives. Journal Traditional Chinese Medicine. 2: 31-38, 1982b.

4. Guo XB, Fu LC, Yu CG, Jian HX, Li GQ. A study on tolerance and toxic side-effects of artesunate intravenous injection on healthy volunteers clinical trials phase I. Clinical trials on Qinghaosu and its derivatives 1:3943,1990.

5. Kohn A, Serapião CJ, Katz N, Dias EP. Ação da oxamniquine sobre o Schistosoma mansoni em camundongos experimentalmente infectados. Revista Instituto Medicina Tropical de São Paulo 21:217-227, 1979.

6. Pellegrino J, Katz N. Experimental chemotherapy of Schistosomiasis mansoni. Advance Parasitology 6:233290, 1968.

7. Qichao Y, Weizhi S, Rei L, Jun G. The antimalarial effect of artesunate on animal models. Journal Traditional Chinese Medicine 2:99-103, 1982.

8. Shuhua $\mathrm{X}$, Catto BA. in vitro and in vivo studies of the effect of artemether on Schistosoma mansoni. Antimicrobial Agents Chemotherapy 33:1557-1562, 1989.

9. Snedecor GW, Cochran WG. Statistical Methods 6th editora lowa. lowa University Press, 1977.

10. Yue WJ, You JQ, Mei JY. Effects of artemether on 